\title{
Analysis of Conductor Displacements in the Coil of the LHC Main Dipole by Speckle Interferometry
}

\author{
P. Ferracin, P. Rastogi, W. Scandale, E. Todesco, and P. Tropea
}

\begin{abstract}
Magnetic field quality in superconducting magnets mostly depends on conductor position in operational conditions (under pressure, at 1.9 K). For the case of the LHC main magnets, the conductor layout must agree with the nominal design within less than $0.05 \mathrm{~mm}$ to met the field quality specifications. Finite element models are a numerical tool to forecast loads and deformations of mechanical structures, and can be used to evaluate conductor displacements. To verify the FEM response at room temperature, we made displacement measurements using speckle interferometer on a short sample of the dipole coils. Experimental results are compared with the numerical calculations, allowing a stringent test of the most critical features of the FEM (interfaces between different materials and coil properties).
\end{abstract}

Index Terms-Finite element model, speckle interferometry, superconducting coil, superconducting magnet.

\section{INTRODUCTION}

$\mathbf{I}$ N THE Large Hadron Collider (LHC), a new accelerator under construction at Europen Organization for Nuclear Research (CERN), an 8.3 tesla magnetic field will be required to bend particles that are accelerated up to $7 \mathrm{TeV}$. For this purpose, superconducting magnets operating at $1.9 \mathrm{~K}$ have been designed [1]. Magnet imperfections may produce instabilities in proton trajectories, thus limiting the accelerator performance. Beam dynamics requirements impose tolerances on the relative magnetic field imperfections of the order of $10^{-4}$. Since in superconducting magnets field homogeneity mainly depends on the position of the conductors, this implies that tolerances on the geometry of components are less than $0.05 \mathrm{~mm} \mathrm{[2],}$ [3]. Finite element models of the LHC dipole cross-section allow computing the stresses arising inside the dipole during assembly and cool-down [4], taking into account the material properties of the magnet components and the applied loads. The results of the numerical computations can also be used to investigate the impact of the coil deformations and of the fabrication tolerances on the field quality [5]-[7]. The aim of this work is to present an experimental method to verify the

Manuscript received September 24, 2001.

P. Ferracin, W. Scandale, and E. Todesco are with the Main Magnets and Superconductor Group, LHC Division, European Laboratory for Particle Physics (CERN), 1211 Geneva 23, Switzerland (e-mail: \{Paolo.Ferracin; Walter.Scandale; Ezio.Todesco\}@cern.ch).

P. Rastogi is with the Institue de Statique et Structure, Ecole Politecnique Fédérale de Lausanne (EPFL), 1015 Lausanne, Switzerland (e-mail pramod.rastogi@epfl.ch).

P. Tropea was with the MMS group at CERN. She is now with the Laboratoire de Mecanique Appliquee et d'Analyze de Fiabilite, Ecole Politecnique Fédérale de Lausanne (EPFL), 1015 Lausanne, Switzerland (e-mail paola.tropea@epfl.ch).

Publisher Item Identifier S 1051-8223(02)04271-9.

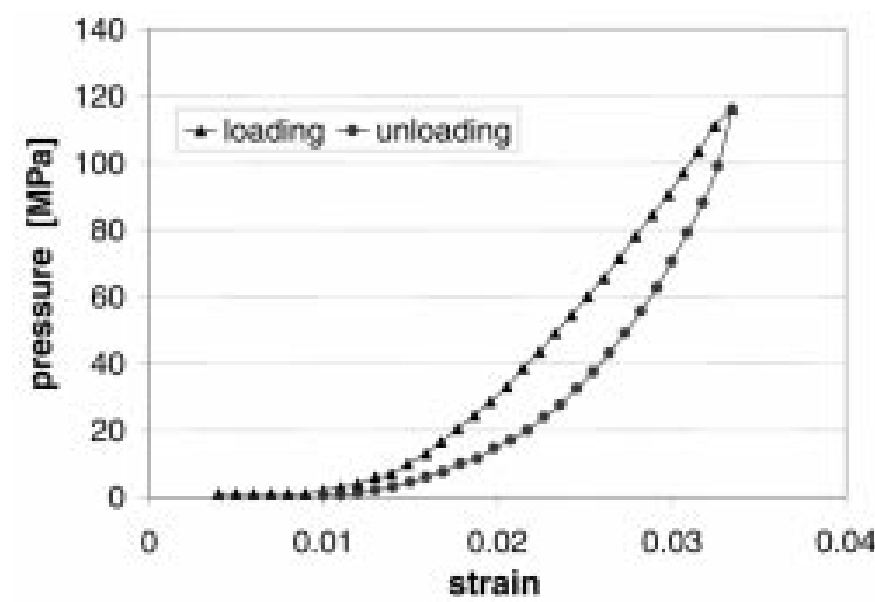

Fig. 1. Experimental loading-unloading/strain curve for conductor stacks.

reliability and the validity limits of the calculations of the LHC dipole model. The displacements of a short sample of the dipole coil are measured by speckle interferometry with a precision better than $0.01 \mathrm{~mm}$, using an optical device implemented at the EPFL (Ecole Polytechnique Fédérale de Lausanne). Then, measurements are compared with FEM results.

\section{THE SUPERCONDUCTING LHC DiPOLE COIL}

Superconducting (Cu/NbTi) cables assembled in blocks and wedge-shaped copper spacers constitute the LHC dipole coils. Blocks are composed of cables that contain cylindrical strands (where NbTi filaments are inserted in a copper matrix) and are insulated using a polyimide tape. This complex composite structure features a nonlinear mechanical behavior under compression [8]-[10].

Moreover, one has an important hysteresis between loading and unloading. This is not negligible since during the assembly and the cool-down the coil undergoes load and unload phases. The pressure-strain curve experimentally measured for a stack of cables is shown in Fig. 1. Stainless steel laminations (collars) clamp the coils; they are assembled with a press to impose a pre-stress of $70 \mathrm{MPa}$ between coil poles and collars at ambient temperature. Between coils and collars and between inner and outer coils, thin layers of different materials (coil protection sheets, shim retainers, shims, ground insulation and quench heaters) are inserted. They are used to give mechanical and electrical protection to the coil. Our reference system is identified by the horizontal and vertical directions of the dipole crosssection $(x, y)$, and by the longitudinal axis of the dipole $z$. 


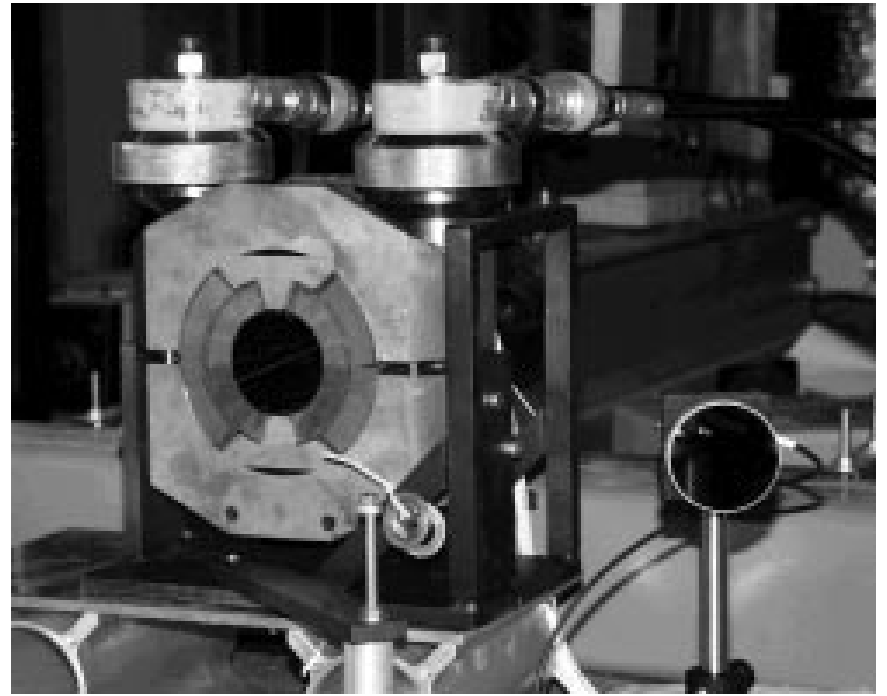

Fig. 2. The mechanical press.

\section{EXPERIMENTAL APPARATUS}

\section{A. The LHC Coil Section and the Hydraulic Press}

A short section $(94 \mathrm{~mm})$ of the dipole coils has been assembled in a small press composed by two stainless steel blocks, molded as the LHC dipole collars on the side in contact with the coils and kept together by two pistons (see Fig. 2). The press is loaded using two hydraulic pistons, whose maximum force is $124.6 \mathrm{kN}$ each. The force is controlled by a manometer with a sensitivity of 3 bars, which corresponds to a stress of $0.2 \mathrm{MPa}$ on the coil mid-plane.

This permits to reach coil stresses up to a maximum of $40 \mathrm{MPa}$ on the coil poles, that is enough to test the most critical features of the mechanical behavior of the coil (nonlinear stress-strain relation of the coil, interfaces between materials). Loads are measured in correspondence of the top and of the bottom arcs of the coils by eight capacitive force transducers [9], whose sensitivity allows sensitivity in pressure of $1 \mathrm{MPa}$ at room temperature.

\section{B. The Optical System}

Speckle interferometry is based on the coherent addition of the light scattered by the object surface with a reference wave. The basic principle of the method resides in the correlation of the speckle patterns at the image plane of the CCD camera employed to detect the speckle irradiance. The displacement undergone by the object is worked out by comparing speckle patterns of two different situations of load and deformation [10], [11]. The subtraction between the two images is performed electronically by a digital frame processor. The resulting intensity distribution is representative of an interferogram in which the in-plane displacement between two successive fringes is given by:

$$
\frac{\lambda}{2 \sin \theta}
$$

where $\lambda$ is the wavelength of the illuminating beams and $\theta$ the angle of the beams with the normal to the image plane. In our setting, we have $\lambda=5145 \AA$ and $\theta=10^{\circ}$, so that two fringes represent a relative displacement of $1.5 \mu \mathrm{m}$.
Since the speckle technique allows to determine displacements along one axis at a time, we have focused our investigations on displacements along the $y$ axis. Indeed, this is the direction of the main mode of deformation of the coil (i.e., an azimuthal compression).

\section{The Finite Element Model}

The finite element code ANSYS ${ }^{\circledR}$ [12] has been used to build a two-dimensional model of our experimental apparatus. Half of the tool is considered, since boundary conditions provide the left-right symmetry but not the up-down symmetry (the press is screwed on the lower part). The press is composed of two blocks of steel. Surface loads are applied in order to model the piston. The steel support is provided by boundary conditions on the lower part of the press. The most critical part of the model is the superconducting coil. They are modeled in terms of their two main components, i.e., blocks of superconducting cables and copper wedges.

To take into account the nonlinear behavior of the coils under compression, the elastic modulus of the blocks is not considered constant. Indeed, the loading part of the experimental curve described in Section II is inserted into the model; this can be implemented using a dedicated option of the code [13].

The second critical feature of the model is the interface between the press and the coil, which is filled with thin materials such as in the real magnet. These materials are not explicitly considered, but are modeled in the FEM through contact elements (CONTAC52). Contact elements behave like springs in the normal direction, reacting to a force proportionally to their stiffness. Since in our case the contact elements model layers of different materials, their stiffness takes into account the elastic modulus of these materials. Contact elements also allow modeling friction, as shown in [14].

\section{EXPERIMENTAL RESULTS}

\section{A. Test Procedure and Results}

Differences in displacements are measured by speckle interferometry in correspondence of two successive load cases, with a stress increment of about $0.8 \mathrm{MPa}$, measured on the manometer. Speckle patterns are acquired in correspondence of the two loads and an image showing level curves of equal displacements is issued. Two consecutive level curves correspond to a difference in displacements along the $y$ axis of $1.5 \mu \mathrm{m}$. We tested the coil behavior for four different values of the initial azimuthal stress in the range of 10 to $40 \mathrm{MPa}$, each time performing a stress increase of $0.8 \mathrm{MPa}$.

In Figs. 3 and 4 the $10 \mathrm{MPa}$ and the $40 \mathrm{MPa}$ load cases are shown. ANSYS ${ }^{\circledR}$ simulations, on the right part of the figures, show equal displacement lines, whose distribution has to be compared with the measured fringes of the speckle interferometry, on the left. As shown in Table I, resuming data relative to all the load cases, there is a good agreement between experimental and numerical simulation results. Moreover, the pattern of the fringes is also well reproduced. In particular, we notice that speckle fringes are not continuous between inner and outer coils. This depends on the sliding of the coils. Contact elements, used in the model to represent these layers of materials, well reproduce this behavior. 

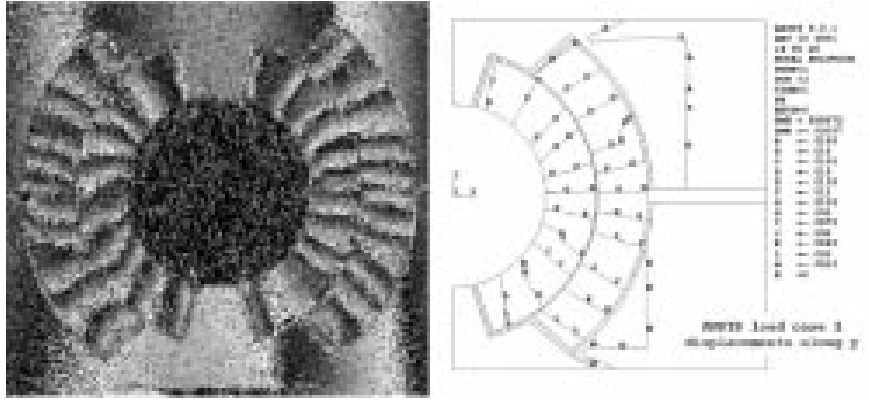

Fig. 3. Speckle interferometry image and ANSYS ${ }^{\circledR}$ displacements for the first load case.
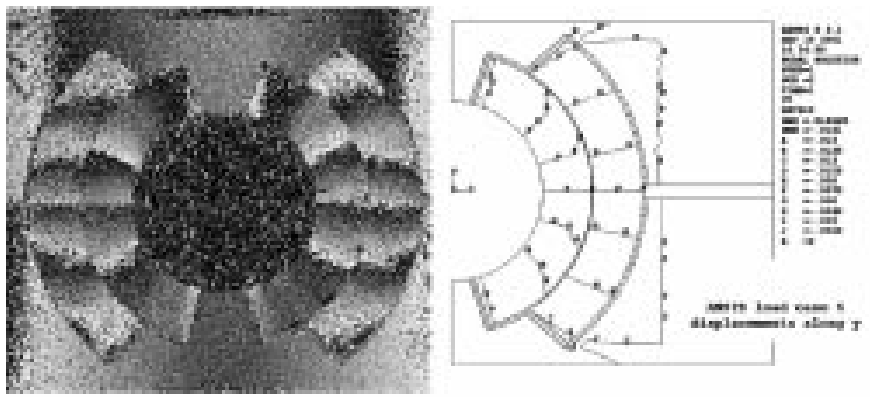

Fig. 4. Speckle interferometry image and ANSYS ${ }^{\circledR}$ displacements for the fourth load case.

It can be also pointed out that the total number of fringes decreases as the applied load increases from $10 \mathrm{MPa}$ (Fig. 3) to $40 \mathrm{MPa}$ (Fig. 4). Since the load step applied during optical measurements is the same, the different number of fringes confirms that the coil has a nonlinear behavior, as found in mechanical tests (see Fig. 1). Finally, we remark that the agreement between interferometry and FEM is worst for higher pressure load cases, where ANSYS ${ }^{\circledR}$ gives one or two fringes more than the speckle interferometry measurements. Indeed, it is possible to improve the agreement by introducing some friction in the contact elements of the FEM. A parametrical analysis has been done and a friction coefficient equal to 0.3 has been chosen. This value is not based on experimental data: in fact the determination of the friction coefficient is not trivial, since one has several layers of different materials to model. All the cases have been re-evaluated and an improved agreement has been found (see Table II): the shape and distribution of the equal displacement lines computed for the high-pressure cases result more similar to the measured ones.

\section{CONCLUSIONS}

We have analyzed displacements of the coil cross section of the LHC dipole under different loads using speckle interferometry and a finite element method. Due to the composite structure of the coil, its mechanical behavior is not trivial. We analyzed displacements along the $y$ axis, with pressures ranging from 10 to $40 \mathrm{MPa}$, and with differential displacements corresponding to 5 to $15 \mu \mathrm{m}$. We found a good quantitative agreement between the displacements measured with our experimental apparatus and the finite element results. In particular, the contact elements used to model the interfaces between the coils and the press provide correct estimates of the sliding between the two materials.
TABLE I

SPECKLE INTERFEROMETRY FRINGES AND EQUAL DISPLACEMENT LINES FROM THE FEM

\begin{tabular}{ccccc}
\hline LOND CASE & \multicolumn{2}{c}{ [SWER COIL FINGES } & \multicolumn{2}{c}{ CUTER COLL. FRINGES } \\
[MPN] & SFECKI. & ANSYS & SEECKLF & ANSYS \\
\hline $10-11$ & 10 & 11 & 11 & 11 \\
$20-21$ & 8 & 9 & 9 & 9 \\
$30-31$ & 6 & 8 & 8 & 8 \\
$40-11$ & 5 & 3 & 5 & 3 \\
\hline
\end{tabular}

TABLE II

SPECKLE INTERFEROMETRY FRINGES AND EQUAL DISPLACEMENT LINES FROM THE FEM WITH FRICTION IN CONTACT ELEMENTS

\begin{tabular}{ccccc}
\hline LOAD CASE & RNEER COIL FINGES & \multicolumn{2}{c}{ CUTER COLL FRINGES } \\
[MFN] & SFECK1.F & ANSYS & SPECKLE & ANSYS \\
\hline $10-11$ & 10 & 11 & 11 & 11 \\
$20-21$ & 8 & 9 & 9 & 8 \\
$30-31$ & 6 & 7 & 8 & 7 \\
$40-41$ & 5 & 5 & 5 & 5 \\
\hline
\end{tabular}

The patterns of fringes of equal displacements of the finite element model and of the experimental measurements well agree in the analyzed cases.

Moreover, we observed an increased rigidity of the coil for higher loads, that agrees with the experimental stress-strain curve derived from cable stack measurements used to model the coil in the finite element code. Introducing a friction between the surfaces in contact, we improved the model and we obtained a very good quantitative agreement between experimental and numerical results.

\section{ACKNOWLEDGMENT}

The authors would like to acknowledge I. Vanenkov, M. Parent, E. Magnani, and M. Cornelis for the help in the design and fabrication of the experimental tools; and J. Trachez and R. Delez for important contribution to the development of the measurements. They thank A. Devred, S. Sgobba, and V. Frigo for useful discussions and comments.

\section{REFERENCES}

[1] "The large hadron collider, conceptual design," in CERN/AC/95-05, October 1995.

[2] A. Devred, T. Ogitsu, and K. Tsuchiya, "Investigation of wire motion in superconducting magnets," IEEE Trans. Magn., vol. 27, pp. 2132-2135, 1991.

[3] D. Bein, J. Zbasnik, J. Bolger, B. Firey, B. Rice, B. Crane, G. Rabaey, J. Wesick, and L. Morgan, "Utilization of gamma ray inspection system for tomographic imaging and dimensional analysis of complete model magnet cold masses and collared coil sections," in Supercollider 4. New York: Plenum Press, 1992, pp. 225-233.

[4] P. Ferracin, W. Scandale, E. Todesco, and D. Tommasini, "Modeling of the coil prestress losses during cool-down in the main LHC dipole," IEEE Trans. Appl. Superconduct., submitted for publication.

[5] W. Scandale, E. Todesco, and P. Tropea, "Influence of mechanical tolerances on the field quality in the LHC main dipoles," IEEE Trans. Appl. Superconduct., vol. 10, pp. 56-59, 2000.

[6] P. Ferracin, W. Scandale, E. Todesco, P. Tropea, and I. Vanenkov, "Modelization of the thermo-mechanical structure of the main LHC dipole and its influence on field quality," in 7th European Particle Accelerator Conf., Vienna, June 2000, pp. 330-332. 
[7] P. Ferracin, et al., "Impact of coil deformations on field quality in the large hadron collider main dipole," IEEE Trans. Appl. Superconduct., submitted for publication.

[8] A. Devred, M. Durante, C. Gourdin, M. Reytier, and P. Vedrine, "Characterization of the thermo mechanical behavior of insulated cable stacks representative of accelerator magnet coils," in DAPNIA-STCM-00-23, Dec. 2000.

[9] N. Siegel, D. Tommasini, and I. Vanenkov, "Design and use of capacitive force transducers for superconducting magnet models for the LHC," in 15th Int. Conf. Magnet Technology, Beijing, China, 1997, pp. 54-58.

[10] P. K. Rastogi, Trends in Optical Non-Destructive Testing and Inspection. Amsterdam: Elsevier, 2000, pp. 171-178.
[11] _- Optical Measurements Techniques and Applications. London: Artech House, 1997, pp. 111-150.

[12] "ANSYS user's manual for revision 5.5," Swanson Analysis System Inc., Houston.

[13] P. Ferracin, E. Todesco, and P. Tropea, "Modeling of the nonlinear elasticity curve of the LHC dipole coils with the finite elements," in CERN LHC-MMS Internal Note 2000-17, 2000.

[14] M. Bajko, P. Fessia, and D. Perini, "FEM computations concerning the effect of friction in two LHC main dipole structures," IEEE Trans. Appl. Superconduct., vol. 10, pp. 81-84, 2000. 